\title{
The SHADES neutron detection array
}

\author{
Chemseddine Ananna ${ }^{1, *}$, David Rapagnani ${ }^{1}$, Andreas Best $^{1}$, Antonino Di Leva ${ }^{1}$, and Gian- \\ luca Imbriani $^{1}$ \\ ${ }^{1}$ University of Naples "Federico II", Via Vicinale, 80145 Napoli, Italy
}

\begin{abstract}
The detection of neutrons in low energy astrophysics poses two main problems: low cross section measurement requires high detection efficiency and ideally, beam-induced and external neutron backgrounds should be identified through an energy sensitive detector. In recent years, capture-gated neutron spectroscopy has found new opportunities to grow through new materials and better availability of modern data acquisition systems. In this work we will present the design, expected capabilities and preliminary characterization of the hybrid neutron detector array SHADES. Its purpose is the first direct measurement of the reaction ${ }^{22} \mathrm{Ne}(\alpha, \mathrm{n})^{25} \mathrm{Mg}$ in the Gamow window for s-process nucleosynthesis with LUNA-MV in the deep underground environment of the LNGS.
\end{abstract}

\section{Introduction}

The ${ }^{22} \mathrm{Ne}(\alpha, \mathrm{n})^{25} \mathrm{Mg}$ reaction is not only one of the two main neutron sources feeding the main s-process but it also is the main source of neutrons available for the weak s-process during the AGB phase for massive stars $\left(M>9 M_{\odot}\right)$. It is, therefore, crucial for the study of the stellar nucleosynthesis of the heavy elements in the universe, particularly those with an atomic mass $60<\mathrm{A}<90$. As of now, only upper limits are known for this reaction's cross section at the resonance energy of $E_{\alpha}=832 \mathrm{keV}\left(J^{\pi}=2^{+}\right)$. Nevertheless, other states do exist below this energy [1-3] but their contribution to the total cross-section hasn't been measured yet because of the low cross section $[1,4,5]$.

\section{Technical challenges}

The ${ }^{22} \mathrm{Ne}(\alpha, \mathrm{n}){ }^{25} \mathrm{Mg}$ has a negative $\mathrm{Q}$-value of $\mathrm{Q}=-478 \mathrm{keV}$, therefore, neutron energies extend down to values that are very low. This represents a first challenge to overcome because the detection and measurement of such low energy neutrons is very difficult.

The measurement of the low cross section requires a high detection efficiency and suppression of external and beam induced background from reactions with impurities like Boron and ${ }^{13} \mathrm{C}$. In the meanwhile, the external neutron background should be identified and discriminated against through an energy sensitive detector, in addition to a passive shielding.

To overcome the aforementioned challenges, the SHADES project (ERC-StG-2019 \#852016) is aiming to perform a high sensitivity measurement, lowering the experimental

\footnotetext{
*e-mail: chemseddine.ananna@unina.it
} 


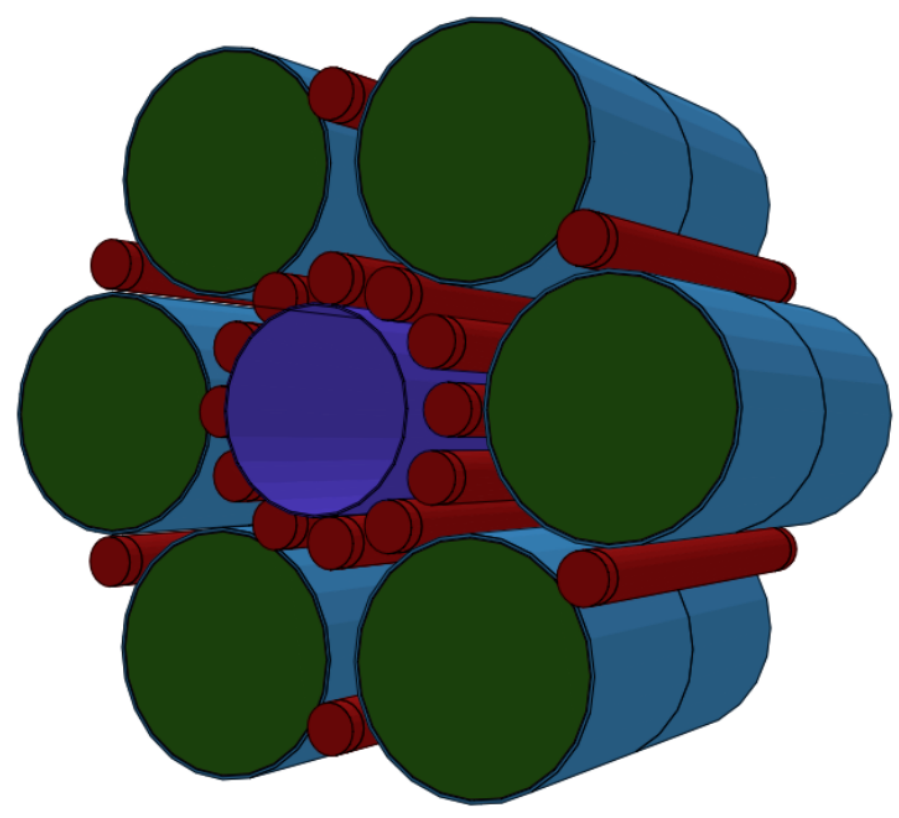

Figure 1. The SHADES detection array

background by going inside the deep underground facility of the Gran Sasso National Laboratory (AQ - Italy) and employing coincidences (and anti-coincidences) between two neutron detector types. The expected background reduction is more than 3 orders of magnitude over the surface lab one.

The new LUNA-MV accelerator [6] will provide a high-current $(\approx 500 \mu \mathrm{A})$, high-stability $\alpha$ beam that will be impinging on a recirculating extended gas target, allowing for a long and interruption-free measurement campaign.

\section{Detection setup}

The SHADES detector array consists of a 12 EJ-309 liquid scintillators ring in between two ${ }^{3} \mathrm{He}$ counters rings of 12 and 6 units, respectively surrounding a recirculating, windowless gas target (see Fig.1). This combination of multiple detector types will allow a better discrimination against both beam-induced and naatural room background by imposing that only events detected in both are considered and by employing cuts on the neutron energy.

The whole detection array will be placed inside borated polyethylene (see Fig. 2 to further reduce the external neutron background.

As a hybrid system, each component of the SHADES detector has a different but complementary task to achieve during the measurement. The liquid scintillators moderate the reaction neutrons and register the recoil energy. When a thermalized neutron enters one of the ${ }^{3} \mathrm{He}$ counters, it will be detected thanks to the high cross section of the reaction ${ }^{3} \mathrm{He}(\mathrm{n}, \mathrm{p})^{3} \mathrm{~T}$. . For the low-energy neutrons coming from the reaction ${ }^{22} \mathrm{Ne}(\alpha, \mathrm{n})^{25} \mathrm{Mg}$, the ${ }^{3} \mathrm{He}$ detector acts as a counter with a total detection efficiency of about $15-20 \%$. The liquid scintillators are not sensitive to neutrons of these energies but will be very useful as anticoincidence detectors: higher-energy neutrons from beam-induced background reactions like ${ }^{10} \mathrm{~B}(\alpha, \mathrm{n}){ }^{13} \mathrm{~N}(\mathrm{Q}$ 


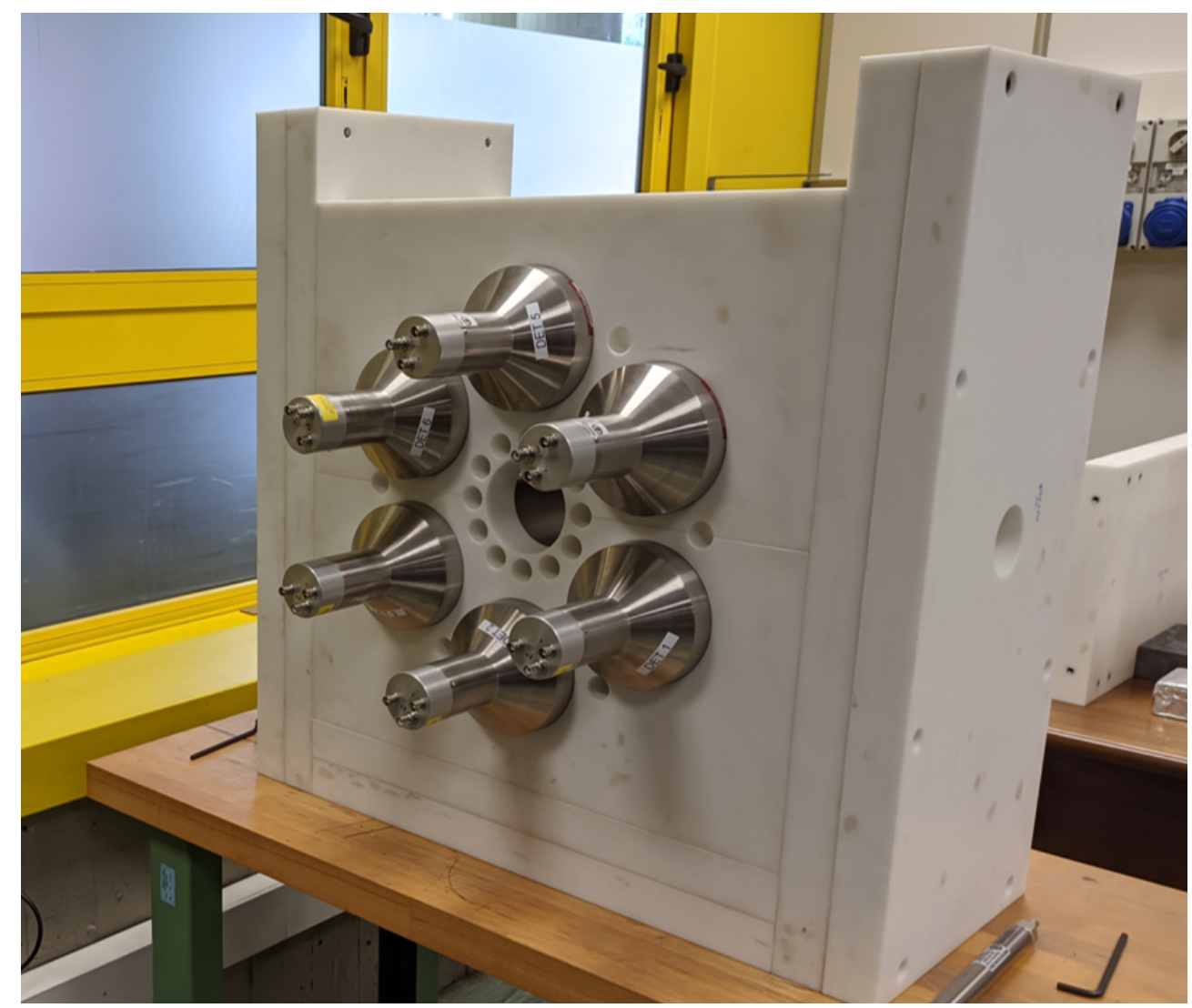

Figure 2. Mounting of the Shades detection array inside the borated polyetylene shielding

$=1059 \mathrm{keV}),{ }^{11} \mathrm{~B}(\alpha, \mathrm{n}){ }^{14} \mathrm{~N}(\mathrm{Q}=158 \mathrm{keV})$ or ${ }^{13} \mathrm{C}(\alpha, \mathrm{n}){ }^{16} \mathrm{O}(\mathrm{Q}=2216 \mathrm{keV})$ will be registered in the scintillators and can be identified and removed.

\subsection{Characterization}

A first characterization measurements of the 12 EJ-309 detectors inside a lead castle have been performed in the LNGS deep-underground from the end of July to the beginning of August 2021. This over a month-long run aimed to measure the intrinsic background activity of the detection array. The gathered data is still under analysis, but the first results seem to be very promising. The Pulse Shape Discrimination figure (see Fig.3) presents a good gamma/alpha separation until an acceptable value towards lower energies.

This first run has also allowed to confirm the low gamma background activity $(\approx 45$ counts. $\mathrm{s}^{-1}$ per detector). A very key feature of the LNGS facility heavily needed in the experiment because of the low cross section of ${ }^{22} \mathrm{Ne}(\alpha, \mathrm{n})^{25} \mathrm{Mg}$ reaction.

\section{Outlook}

In the near future, the SHADES team is aiming to better refine the used PSD techniques to get a better alpha/gamma and neutron/gamma separation towards lower energies. The liquid 


\section{PSD VS Energy}

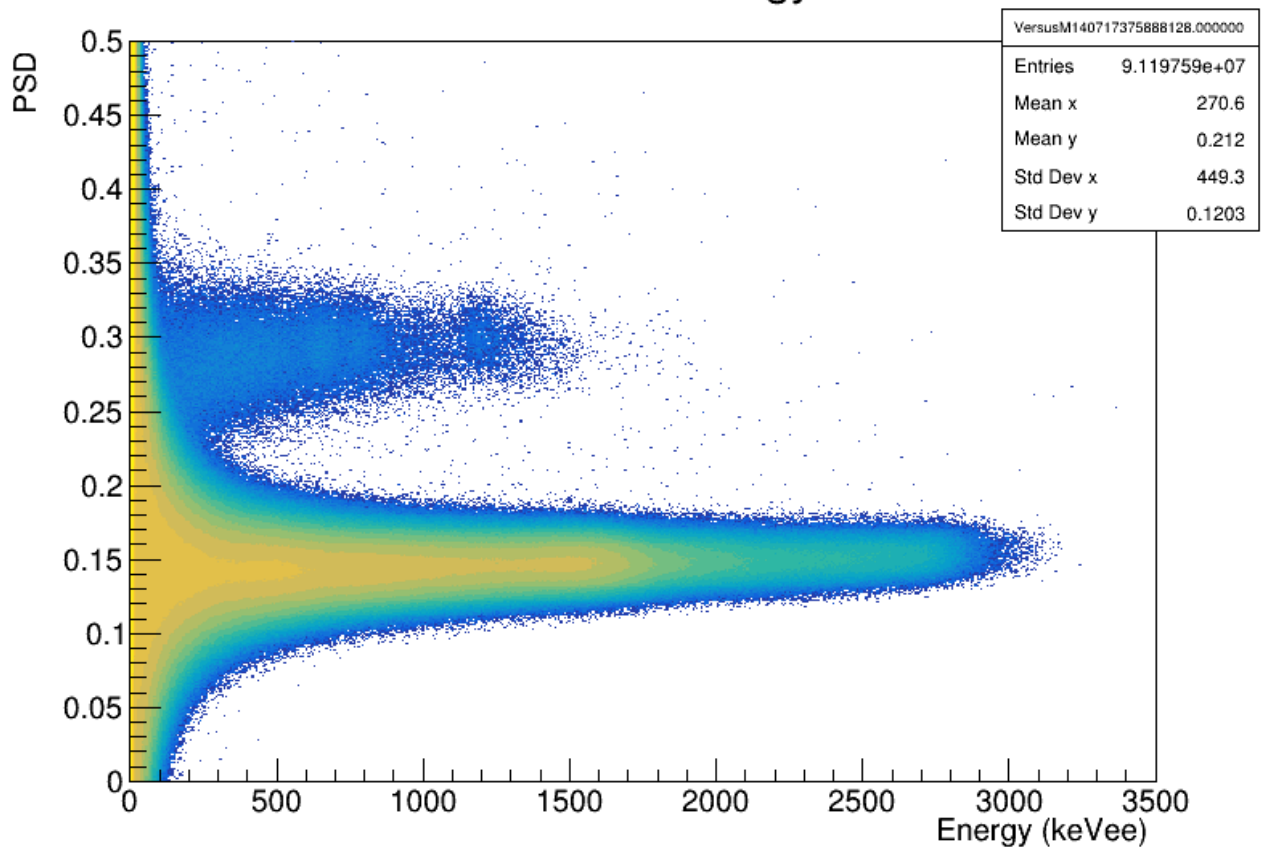

Figure 3. Alpha/Gamma pulse shape discrimination, LNGS, a month-long acquision run using the charge integration method.

scintillator characterization should also be resumed by doing the quenching calibration. The performance of the full array (EJ-309 scintillators $+{ }^{3} \mathrm{He}$ counters) should then be ready to be tested as a whole. The second part of the procedure will consist of the characterization of the ${ }^{22} \mathrm{Ne}$ gas target followed by the first LUNA MV beam trials.

\section{Acknowledgement}

The authors acknowledge funding from the European Research Council (ERC-StG 2019 \#852016)

\section{References}

[1] M. Jaeger, R. Kunz, A. Mayer, J.W. Hammer, G. Staudt, K.L. Kratz, B. Pfeiffer, Phys. Rev. Lett. 87, 202501 (2001)

[2] V. Harms, K.L. Kratz, M. Wiescher, Phys. Rev. C 43, 2849 (1991)

[3] H.W. Drotleff, A. Denker, H. Knee, M. Soine, G. Wolf, J.W. Hammer, U. Greife, C. Rolfs, H.P. Trautvetter, Astrophys. J. 414, 735 (1993)

[4] S. Bisterzo, C. Travaglio, R. Gallino, M. Wiescher, F. Käppeler, Astrophys. J. 787, 10 (2014)

[5] C. Massimi, P. Koehler, S. Bisterzo, N. Colonna, R. Gallino, F. Gunsing, F. Käppeler, G. Lorusso, A. Mengoni, M. Pignatari et al. (n_TOF Collaboration), Phys. Rev. C 85, 044615 (2012) 
[6] A. Sen, G. Domínguez-Cañizares, N.C. Podaru, D.J.W. Mous, M. Junker, G. Imbriani, V. Rigato, Nucl. Instrum. Methods Phys. Res. B 450, 390 (2019) 